\title{
ISCHAEMIC AND POST-ISCHAEMIC NUMBNESS AND PARAESTHESIAE
}

\author{
BY \\ P. W. NATHAN \\ From the Neurological Research Unit of the Medical Research Council, National Hospital, \\ Queen Square, London
}

During the last 10 years much interest has been taken in the sensory and motor phenomena associated with the occlusion of the blood supply to a nerve. As only a sphygmomanometer is needed to carry out the experiment, it seems desirable for all physicians to become acquainted with the disturbances of sensation that ensue, for the experiment reproduces in a short time many of the subjective sensory disturbances for which patients seek medical advice. When this simple method of investigation is carried out on patients, it may bring out abnormalities in function that are too slight to be detected by other clinical methods. The results of investigating certain clinical conditions by occluding and releasing the blood supply to the limbs have been published recently. Gilliatt and Grahame Wilson (1953) used this procedure to establish the diagnosis of the carpal-tunnel syndrome, and later Gilliatt (1955) showed that in patients with spinal cord lesions the time to numbness following occlusion of the circulation provides a good indication of the progress of the condition. Poole (1956a) established the limits of the normal by recording the duration and severity of ischaemic and post-ischaemic paraesthesiae in 93 normal subjects; and he later reported the changes found in these paraesthesiae in polyneuritis (1956b) and in motor neurone disease (1957).

As a result of the work of Lewis, Pickering, and Rothschild (1931), Lehmann (1937), Kugelberg (1944, 1948), Sinclair (1948), Merrington and Nathan (1949), Kugelberg and Cobb (1951), Marshall (1952, 1953), and Cobb and Marshall (1954), it is now known that the following events occur when the blood supply to a nerve or to a certain adequate length of nerve is occluded.

A few minutes after the occlusion has started, the nerve fibres of the $A$ group become hyperexcitable. This increased excitability is sufficient to result in the fibres firing off spontaneously. When this spontaneous firing occurs in certain sensory fibres of the $A$ group, tingling in the cutaneous distribution of the nerve is felt. As the period of occlusion of the circulation is continued, the nerve fibres pass through a stage of normal excitability, and eventually after a stage of decreased excitability reach a stage of complete inexcitability. When this stage is reached, the digits feel numb or "dead"; at the same time the small muscles of the hand or foot become paralysed. In the upper limb this occurs about 25 minutes and in the lower limb about 30 minutes after the onset of the occlusion. On restoration of the blood flow, the nerve rapidly regains its ability to conduct. The fibres of the $A$ group first pass through a stage of increased excitability, longer lasting and far more intense than that occurring during the ischaemic period. At this stage the long motor and sensory fibres of the $A$ group fire off spontaneously. This is manifested with regard to the motor fibres by fasciculation in the muscles supplied by the nerve and even by tetanus; with regard to the sensory fibres spontaneous firing is manifested as paraesthesiae. If at this stage the nerve is stimulated electrically, single impulses are transformed into double or multiple impulses. The work of Bazett and McGlone $(1929,1932)$ and of Merrington and Nathan (1949) shows that the various forms of paraesthesiae are related to the groups of fibres subserving various sensations, namely, the thermal paraesthesiae from discharges of the fibres subserving thermal sensation, tingling from discharges of the fibres subserving touch, pricking from discharges of the fibres subserving pressure in the skin, and pseudo cramp from discharges of the afferent fibres from muscle. Ten to 15 minutes after an occlusion lasting 20 minutes the excitability of the fibres returns to normal; at this time the subject no longer experiences any abnormalities in motor or sensory function. These changes in excitability are more prominent in the large peripheral nerves (median, ulnar, sciatic) than in small nerves. And it seems to be that the increased excitability is more marked in the proximal than in the distal parts of the nerves. It is known that all the changes mentioned occur in the nerves and not in the end-organs. 
FIG. 1.-Circuit diagram of stimulator delivering a spark stimulus.

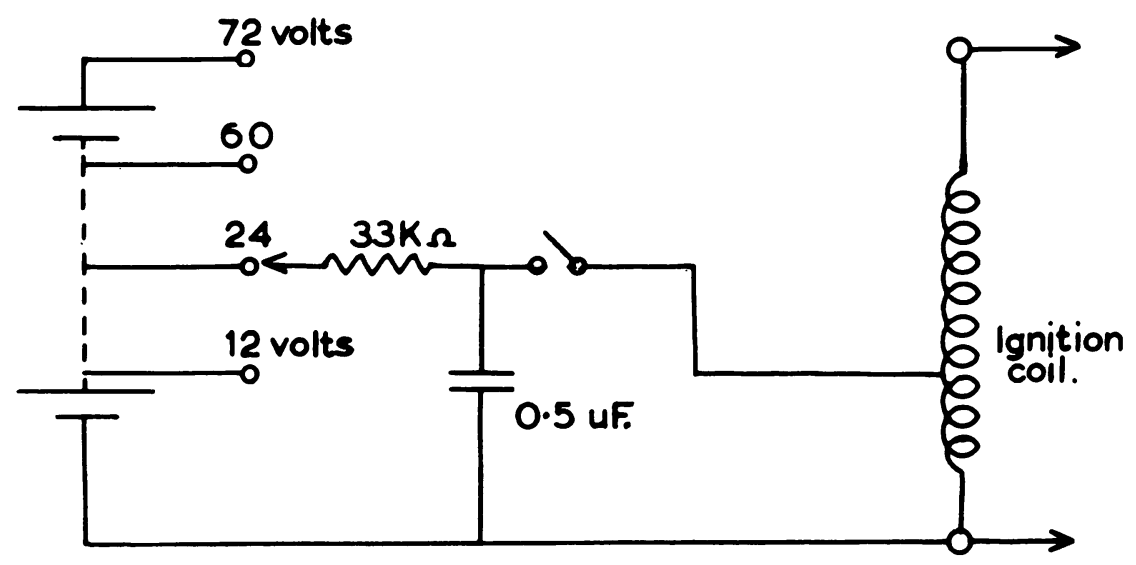

The tingling felt during ischaemia is called "ischaemic tingling " and the paraesthesiae after restoration of the blood supply " post-ischaemic paraesthesiae".

At the times when paraesthesiae occur, interference with the perception of exteroceptive stimuli has been noted. Kugelberg (1944) noticed that when ischaemic tingling is pronounced " the appreciation of touch with a piece of cotton-wool is evidently diminished." Gilliatt and Grahame Wilson (1954) found that during the periods of ischaemic and postischaemic paraesthesiae tactile stimuli applied to the area supplied by the spontaneously discharging nerves are not felt or the response is diminished. A failure to appreciate cutaneous stimuli occurs at the times when the nerve is in a state of increased excitability. It is apparent that an investigation of this phenomenon may throw light on the mechanism of disorders of sensation experienced by patients suffering from a variety of disorders. The purpose of this paper is to report the results of an investigation on the numbness that occurs when nerve fibres are in a state of increased excitability.

\section{Methods of Investigation}

Stimuli of various kinds were applied at intervals of one minute to the dorsal surface of the ultimate phalanx of the big toe, index, or little finger immediately proximal to the nail.

The following methods of stimulation were used:-
(1) von Frey hairs for tactile stimulation which were made of nylon suture thread, and calibrated by being weighed against a balance. The weight in grams at which the hair started to bend was divided by the radius of the hair in millimetres, this figure denoting the "strength" of the hair; (2) the Weber'sche Tastzirkel (usually called in English, a two-point discriminator).

For pain sensibility (3) weighted needles, and (4) a modified version of the instrument designed by Bishop (1943) was made by Mr. H. B. Morton; the circuit diagram is shown in Fig. 1. The stimulus applied by this instrument consists of a spark, the spark gap being about $1 \mathrm{~mm}$. The primary winding of the ignition coil was fed with a charge accumulated in a 0.5 microfarad capacitor charged from the selected input voltage through a 33 kilo-ohm resistor. The switch was in the primary circuit. The voltage used was 60 or 72 volts. The spark which this instrument gives was applied as follows. A screw connected to the output was sunk in a perspex block, which fitted the terminal phalanx; it was fixed on the digit by rubber bands. By raising or lowering the screw, the size of the spark gap could be varied. The advantage of such a stimulator, as Bishop (1944) maintained, is that the electrical stimulus is sharply localized and of shorter duration than any mechanical stimulus; moreover, it causes no deformation of the skin. Applied to the skin of the terminal phalanges, just proximal to the nail, this spark usually gives rise to a sensation of pricking pain. If, instead of this prick, either a slight tap or a pain so slight that it was hardly painful was experienced, then the perspex application was moved a millimetre or so, 
until a place was found where stimulation gave a sharp, painful prick; this sensation was immediate and not delayed. It was possible to show that the impulses set up by this form of stimulation were not transmitted in $C$ fibres. On theoretical grounds alone, the rising time of the spark stimulus is so short that it would be unlikely to give rise to impulses in fibres having such good accommodation as $C$ fibres. It was readily shown, by occluding the circulation to the limb, that the spark stimulus no longer aroused a sensation when only $C$ fibres remain conducting; indeed this stimulus ceased to arouse a sensation before the von Frey hair ceased to do so. Thus it may be concluded that this instrument causes impulses in fibres normally used for the sensation of pricking pain, but not the $C$ fibres.

(5) The electrical stimulation devised by Dawson and reported by Dawson and Scott (1949) and Dawson (1956) was applied through ring electrodes around the fourth and fifth digits of the upper limb; it caused a sensation best described as a slightly painful, heavy blow on the digits.

The stimuli, apart from that using the ring electrodes, were applied in the following manner: The point less than $1 \mathrm{~mm}$. diameter at which the stimulus was applied was marked in ink. The number of affirmative answers to 10 stimulations was noted at each minute. On most occasions all 10 stimuli were applied within the first 30 seconds of the minute. Before each group of 10 stimuli, the subject was warned by the words " Ready ? Now !" The rate of application of the stimuli was random, a rhythm of stimulation being studiously avoided. It was found that provided the stimuli were not applied too rapidly, hallucinations of touch were very rare in most subjects; they did occur, however, when sensibility began to fail completely, after about 20 minutes of occlusion in the upper limb; each hallucination was then scored as a missed real stimulation. The von Frey hairs were applied in such a manner that the distance between the tip of the hair and the skin was about $1 \mathrm{~cm}$. before the hair was lowered on to the skin; the hair was pressed on to the skin until the hair bent, and care was taken to apply the hair on each occasion in as similar a manner as possible.

The two-point discriminator was used with a distance of $2.5 \mathrm{~cm}$. between the points; the results were scored, in order to permit the graphic presentation, as follows: $A=$ correct response with no difficulty in judging; $\boldsymbol{B}=$ doubtful response, with difficulty in judging; $C=$ wrong response or complete inability to judge.

Vascular occlusion was obtained by using the ordinary sphygmomanometer cuff on the arm and a specially designed wide cuff on the thigh. A standard position on each limb was used: the cuff was placed on the thigh with its lower edge 4 to $5 \mathrm{~cm}$. above the upper edge of the patella, and on the arm with its lower edge 1 to $2 \mathrm{~cm}$. above the medial epicondyle.

The skin of the limb was kept at a nearly constant temperature by placing the limb within the field of radiation of heating lamps arranged in an inverted cradle. The skin temperature was monitored by means of a thermojunction.

Electrical stimulation was used by means of the ring electrodes. Records of the nerve action potentials through the skin were, with minor modifications, made in the way described by Dawson and Scott (1949) and Dawson (1956). The compound action potential due to the ascending volley was recorded at two places over the ulnar nerve by two pairs of electrodes: one pair was placed distal to the sphygmomanometer cuff, just above the wrist, and the other pair proximal to the cuff, just above the elbow. An earth connexion was made to a place on the flexor surface of the forearm. In order to obtain adequate relaxation so as to avoid obscuring the induced action potential by artifacts due to muscle action potentials, the subject lay on his back with his upper limb supported on straps slung from a beam. The stimulus on the digits applied by the ring electrodes gave rise to a single synchronous impulse, which, it is known, is conducted in large fibres.

The experiments carried out on healthy subjects are not all given in the paper; a typical one is chosen from a series of similar experiments performed on at least 10 healthy subjects. Experiment 8 is one of three similar experiments.

\section{Experimental Findings}

The phenomenon under investigation is best shown by Experiment 1, illustrated diagrammatically in Fig. 2.

Experiment 1 (Fig. 2). - The stimulus was a von Frey尺 nylon hair No. 3, strength 1 . Before the cuff was placedo on the thigh, the subject, a healthy male aged 32 , waso stimulated 50 times on the dorsum of the big toe, to be $\frac{\mathbb{O}}{O}$ certain that the hair strength was above threshold. Theo period of occlusion was 19 minutes.

As can be seen from Fig. 2, during the phase of ischaemic tingling, the ability to feel the stimulation was very slightly impaired. During the phase of post-ischaemic paraesthesiae, the temporal pattern of disturbance of the ability to feel the stimulus of the hair was found. Within half a minute of releasing the cuff, the stimuli were felt again; in this experiment $7 / 10$ were felt. Three-quarters of a minute after releasing the cuff, post-ischaemic tingling and pricking started, at which time some of the stimulations were still causing a sensation. By the time the post-ischaemic paraesthesiae were fully developed no stimuli with the hair could be felt. Three minutes after releasing the cuff, the appreciation of the stimuli was fully restored; the post-ischaemic paraesthesiae were by this time diminishing, though some tingling remained.

The temporary slight hypoaesthesia accompanying the ischaemic tingling is not easily observed. If the stimulus is too strong, no diminution in the number of correct responses is found; the stimulus strength has to be just threshold in the normal period. The failure to respond to the stimuli is not due to boredom or to lack of attention. If the experiment is carried out again, omitting the application of the cuff, the subject continues to respond to all the stimuli applied throughout every minute of the 22 occupied by the test.

The failure to respond to the stimulation is more 
FIG. 2.-Cuff on thigh. Stimulus, hair of strength 1.
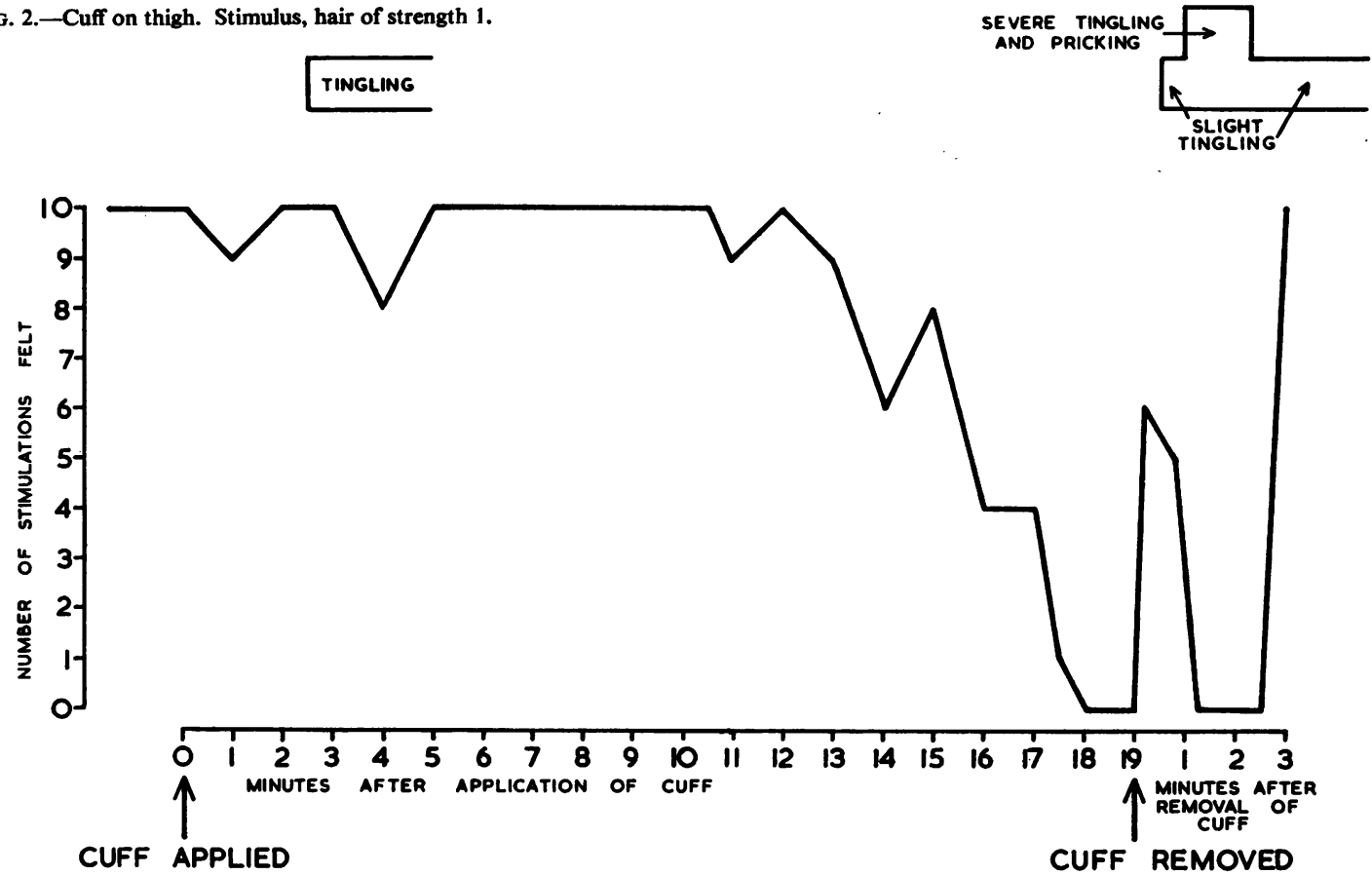

definite during the period of post-ischaemic paraesthesiae; then, even the application of relatively heavy hairs evoked no response.

Although this failure to feel exteroceptive stimulation is only slight in the ischaemic phase among healthy people, in patients with pathological conditions of the nervous system this failure is more pronounced. Experiments on patients with very slight defects of sensibility show the inability to feel the stimulation in a marked form. An example is presented in the next experiment.

Experiment 2 (Fig. 3).-A similar experiment to Experiment 1 was carried out on a patient suffering from meningo-vascular syphilis. In this patient the threshold for touch sensibility was very slightly raised. A stimulus of a nylon No. 1 hair, weight $0.05 \mathrm{~g}$., was applied to the dorsum of the little finger just proximal to the nail; the cuff was in the standard position. A diminution in the number of correct responses occurred at the first minute and continued till the end of the sixth; this was concurrent with ischaemic tingling. The diminution in the response to stimulation was more intense than is found in healthy subjects.

How the diminution in sensation is related to the strength of the stimulus is shown in the next experiment, carried out on a healthy subject.

Experiment 3 (Fig. 4).-Two strengths of stimulus were
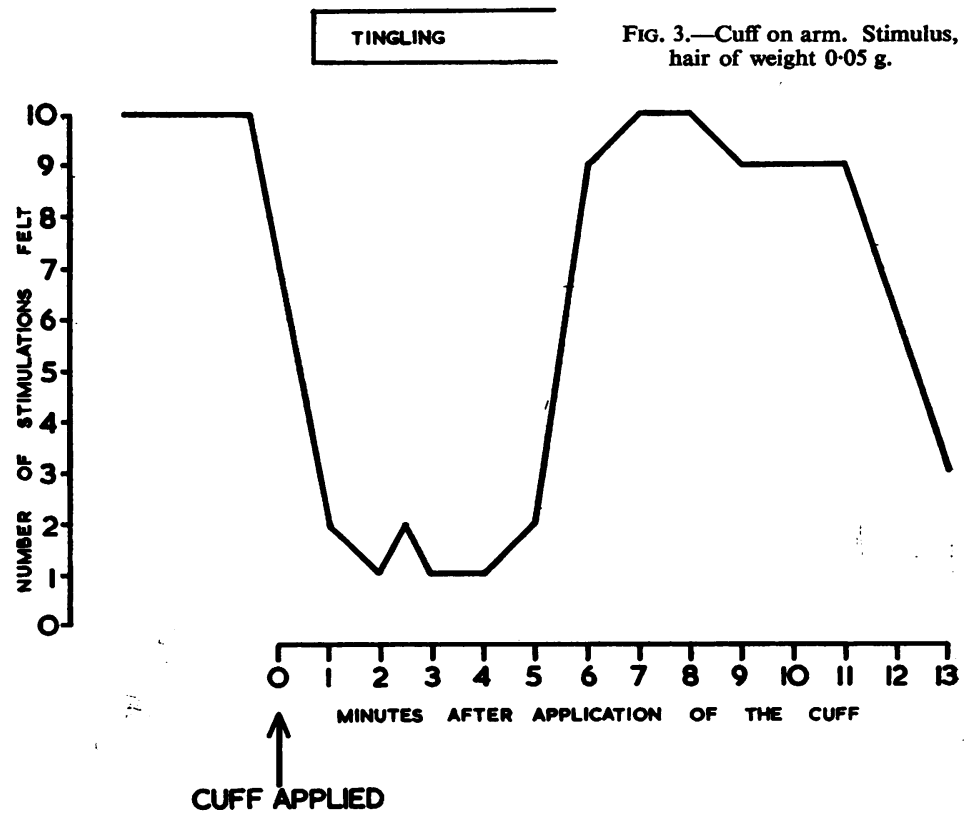


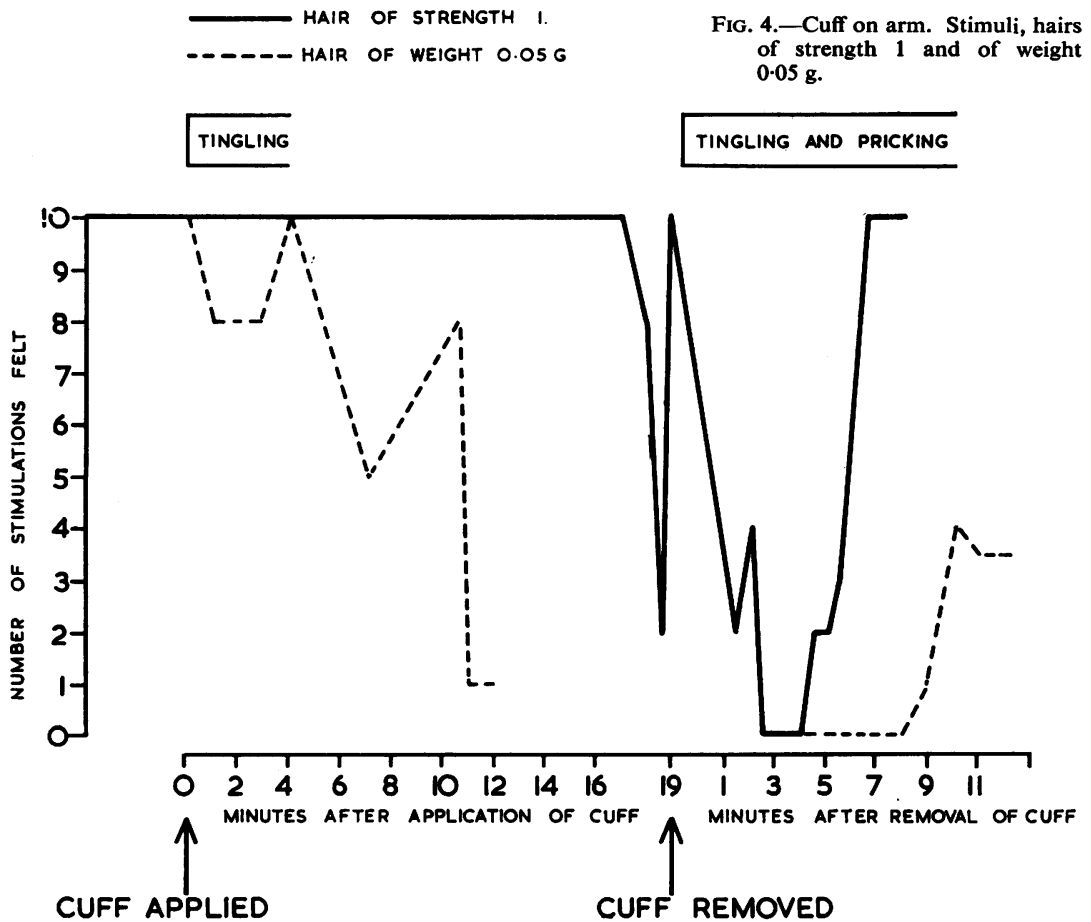

lation to the whole limb, a clamp is applied to a single nerve so as to obstruct its blood supply over a distance of $5 \mathrm{~cm}$. or more, then all the phenomena will be restricted to the distribution of that nerve. If the ulnar nerve is compressed, then ischaemic and post-ischaemic paraesthesiae will be felt only within the cutaneous distribution of that nerve. The touch stimuli applied to the two sides of the fourth digit, one being within the ulnar and the other within the median territory, give rise to different experiences during the periods of paraesthesiae; all those applied to that half of the digit supplied by the median nerve continue to be felt, whereas a percentage of those applied to the other half of the digit are missed. Though the two points stimulated be only $\frac{1}{2} \mathrm{~cm}$. apart, the subject is able to appreciate very light touches on the median half of the fourth digit while he experiences showers of paraesthesiae on the ulnar half of the digit.

The next problem was to find out if this obliteration of sensation is limited to that provoked by very slight stimulation with nylon hairs. It was found that the alteration of sensation is not merely limited to touch caused by the von Frey hairs. For, during the post-ischaemic phase, the sensation induced by rubbing the fingers together feels altered, blurred, or, in the words of most patients, it feels " numb". The following experiment also shows that there is a disturbance of those forms of sensibility needed in discriminating two points.

Experiment 4 (Fig. 5). - The cuff occluding the circulation was placed on the thigh of a healthy male aged 30 . Two forms of stimuli were applied to the dorsum of the second digit, namely, a nylon No. 3 hair and the twopoint discriminator with the points $2.5 \mathrm{~cm}$. apart. It was found that during the ischaemic tingling the subject was unable to feel all the stimuli with the von Frey hair and was unable also to discriminate the two points with certainty.

It may thus be concluded that heavier touch sensibility is also obliterated during the time of the 
paraesthesiae. This was also shown on innumerable occasions when heavier von Frey hairs were used. Although the subjects continued to feel all such stimuli, they noticed that the sensation aroused was less distinct during the times of paraesthesiae.

It was decided next to find out if during the periods of paraesthesiae there is alteration in the threshold for painful stimuli.

Experiment 5 (Fig. 6).-The stimulus was the "sparker" mentioned under " Methods". The voltage was 60 ; the perspex applicator was fixed on the tip of the big toe of a healthy male aged 40. The pressure in the cuff was kept raised for 13 minutes. The number of stimuli felt is plotted in Fig. 6. However, in experiments where a painful stimulus is used, it is more important to record the sensations of the subject. The subject reported as follows:

At 3 min., "The stimulus feels about half as strong as previously."
Fig. 5.-Cuff on thigh. Stimuli, hair of strength 1 , and two-point discriminator, the points at $2.5 \mathrm{~cm}$. apart. In the scoring, $A=$ correct response, with easy discrimination. $B=$ doubtful response, with difficulty in dis-
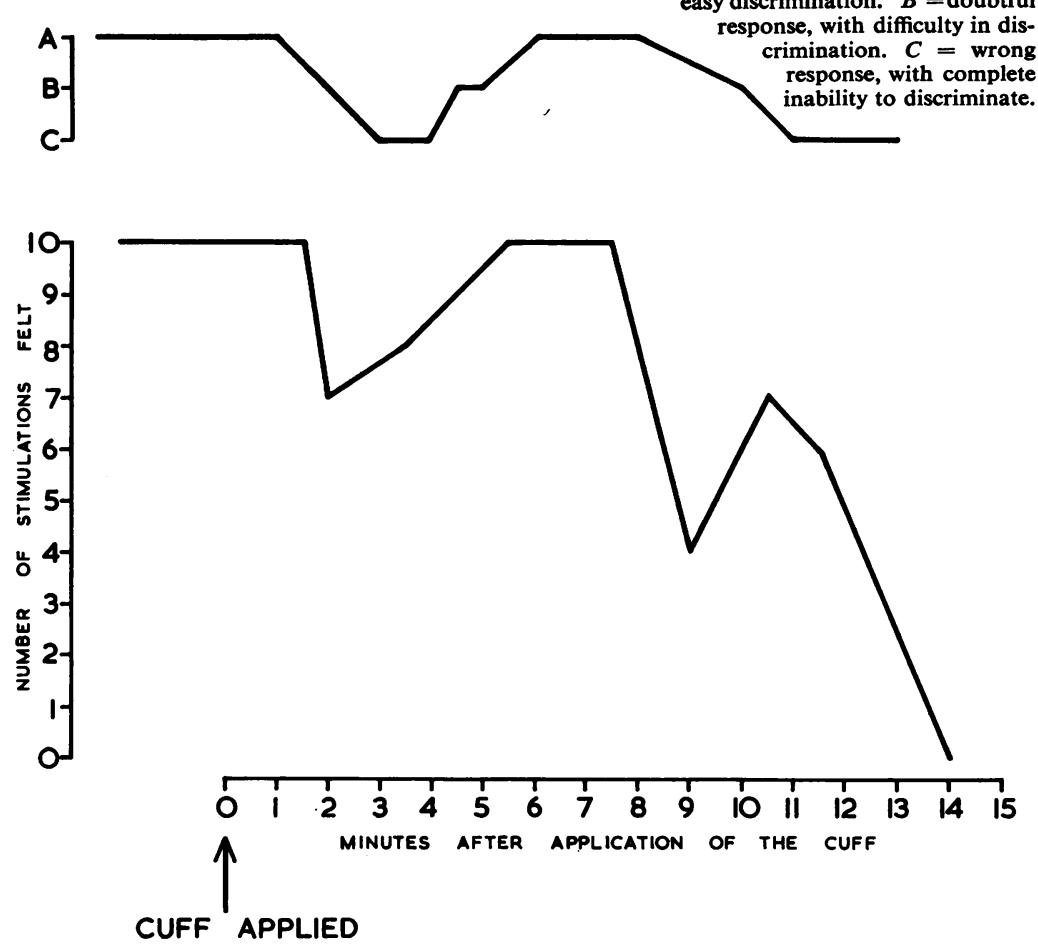

Fig. 6.-Cuff on thigh. Stimulus, sparker, voltage 60 .

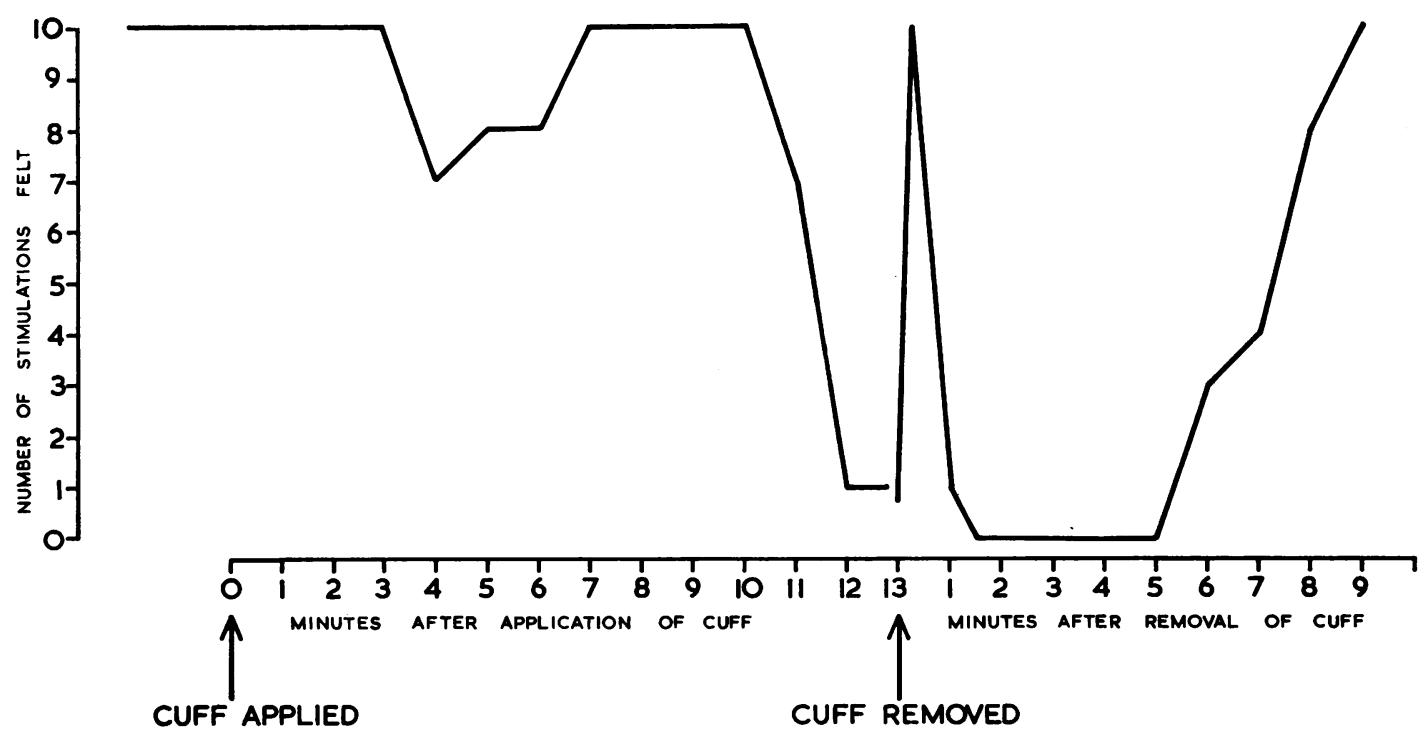


At 5 min., "It is still very weak." The two stimulations that were not felt caused no sensation of any kind.

At 6 min., "They are quite unlike the original pricks."

At 7 min., "Now they feel the same as they did originally."

At 8 min., "They are exactly the same as they were originally."

At 9 min., " The stimuli are slightly weaker, but they are still painful pricks."

At 10 min., "Very weak, but still painful pricks."

At 11 min., three stimuli caused no sensation.

During the post-ischaemic phase, all the stimuli were felt half a minute after release of the cuff; of these, the first five seemed to the subject to be about half the full strength, the second five of full strength. Then, until five minutes after release of the cuff, no stimuli were felt; nine minutes after release, although the stimuli were felt, the sensation aroused was still about half normal.

It will be seen that the plotted curve closely resembles the curves obtained when a light touch stimulus is used. A difference is that the inability to feel the painful stimulus during the post-ischaemic phase lasted a long time, as did the paraesthesiae.

It is not always possible to discriminate objectively an alteration in sensation when subjectively the alteration may be marked; this is especially likely when the sensation is that of pricking pain. Although, in the next experiment, 10 out of 10 stimuli from the " sparker" continued to be felt as pricking pain throughout the period of ischaemia, the quality of the sensation changed. This change in quality occurred at a time when in some subjects a quantitative change was demonstrable.

Experiment 6.-The input voltage of the " sparker" was 72. The cuff was placed on the thigh of a healthy male aged 32. The stimuli were applied to the dorsum of the second digit. The subject reported as follows:

At 3 min., "Some of the sting has gone out of the pricks."

At 4 min., "It feels the same; some of the sting has gone."

At 5 min., "Not all the stimuli have the same intensity."

At 6 min., " They feel definitely duller."

At 7 min., " Now they all feel sharper. They feel about the same as they did before the cuff was put on."

At 8 min., "Very slightly duller; but not as dull as they were earlier on, at about three minutes."

At 10 min., " Duller, less sharp in quality."

At 11 min., " Toes are slightly numb when rubbed."

At 12 min., "This numbness is definite. Now some of the spark stimuli are quite definitely not pricking at all; they have become touches."

Half a minute after release of cuff, nine stimuli were felt as touch, one as prick.

One and a half minutes after release, the paraesthesiae are marked. "I cannot really tell if the stimuli are like pricks or touches."

At $2 \mathrm{~min}$. after release, two stimuli felt as touch, eight not felt at all.
At $3 \mathrm{~min}$. after release, two stimuli felt as very slight pricks, eight felt as touch.

At $5 \mathrm{~min}$. after release, all 10 felt as the same pricking sensation as at the beginning of the experiment.

This experiment illustrates the fact that, when all the stimulations arouse a sensation, during the periods of paraesthesiae the quality and intensity particularly of the sensation is altered.

Also within the modality of pain, the heavier of two stimuli may be felt at the time of the paraesthesiae, while the lighter is not. This is illustrated in the following experiment.

Experiment 7 (Fig. 7).-The stimuli were pins weighted with 5 and $20 \mathrm{~g}$. The cuff was on the thigh of a healthy male, aged 36, for 22 minutes. The results for the period after release of the cuff are shown in Fig. 7. There is the usual obliteration of sensation during the period of intense paraesthesiae; the responses to stimulation with the $5 \mathrm{~g}$. pin are lost far longer than those with the $20 \mathrm{~g}$. pin.

All the experiments reported so far have been repeated, using a clamp to occlude the circulation only to a certain length of a single nerve. All the phenomena described above occurred, though the changes were of a slighter degree. It may therefores $\vec{N}$ be concluded that the alteration of the sensations of light touch, of heavy touch or skin pressure $\mathbb{D}$ and of pricking pain, are not the result of ischaemia
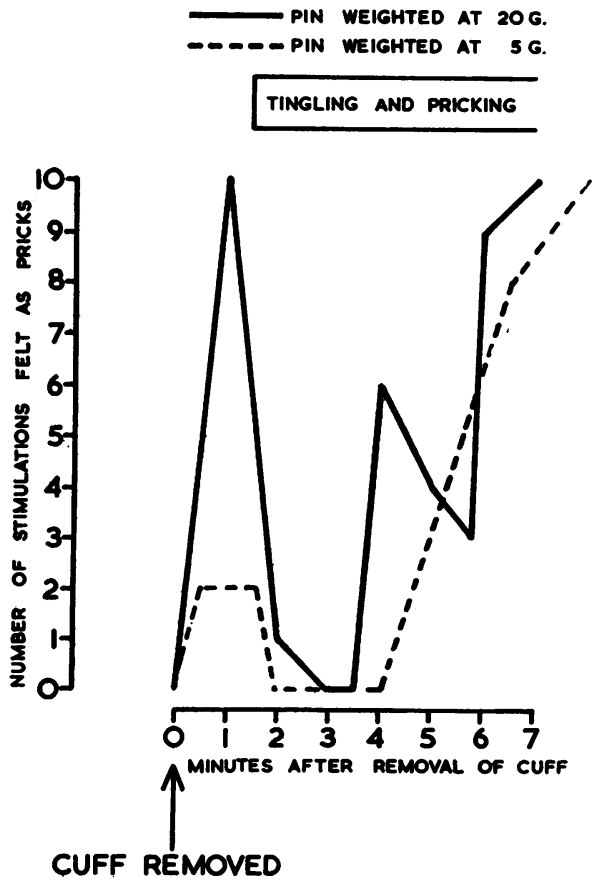

Fig. 7.-Cuff on thigh. Stimuli, pins weighted at 5 and $20 \mathrm{~g}$. 
of the end-organs* but of the nerve itself.

In considering the mechanism causing the raising of the threshold, it is unlikely that it is due to an inability of the nerve fibres to conduct impulses, for the period of ischaemic tingling is followed by a period of perfect conduction during which all stimuli are felt. Again, during the post-ischaemic phase, on release of the vascular occlusion, all or almost all of the stimuli are felt, showing an apparent recovery of the nerve's ability to conduct, and yet a few seconds later, when recovery would be thought to have progressed, the threshold to stimulation is raised again.

Two different explanations of this raised threshold to sensory stimulation may be considered. One explanation offered is that the impulses caused by the test stimulus cause insignificant effects within the central nervous system already bombarded by a

"The term " end-organ" is used not to imply any histologically demonstrable structure. Physiologically there is reason to believe that the termination of a nerve (the receptive element) has special properties, different at least quantitatively from those of the nerve itself (the conducting element). The term is used here to mean this physiological aspect of the receptive element. profusion of impulses arriving from the nerve which is discharging spontaneously. This overwhelming of the signal could be occurring at some level in the central nervous system, yet it need not be conceived as necessarily involving consciousness. Another explanation offered is that the impulses caused by the test stimulus travelling orthodromically are blocked by antidromic impulses arising spontaneously within the nerve fibres in the region of ischaemia. These two groups of impulses travelling towards each other from opposite ends of the fibre could extinguish each other. Whether the impulses due to peripheral stimulation would get through to the central nervous system or not would depend on the number generated in a unit of time and the number of spontaneous impulses generated; eventually some peripherally arising impulses would get through the region of spontaneously firing impulses, if the former are arising faster; and conversely, if they arise less frequently than those generated spontaneously in the hyperexcitable nerve fibres, then they would be obliterated before they reached the central nervous system.
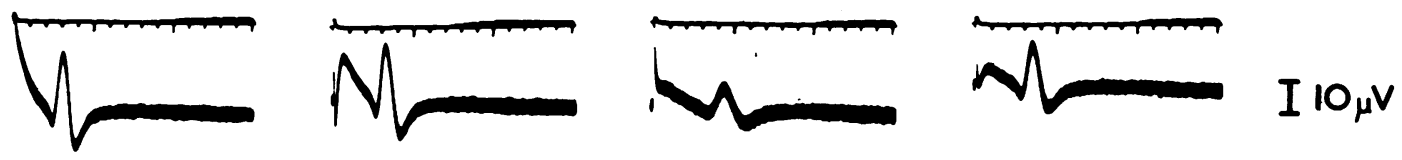

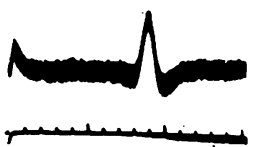

A

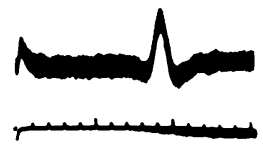

B

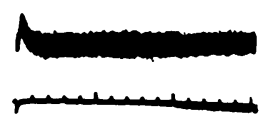

C
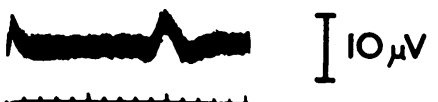

D

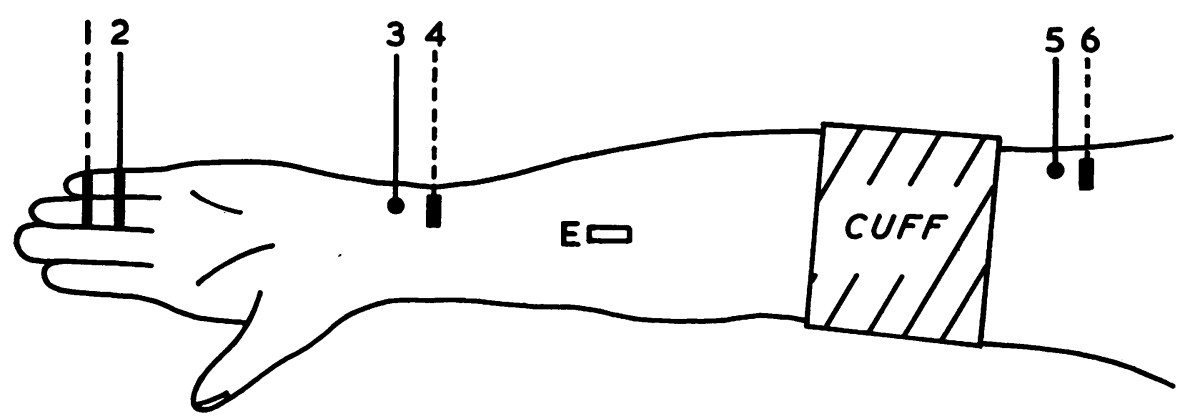

FIG. 8a.-Superimposed tracings of the action potentials produced by stimulating the fourth and fifth digits recorded over the ulnar nerve at two places. The upper tracing shows the centripetal volley recorded at electrodes 3 and 4 , and the lower tracing the volley recorded at electrodes 5 and 6 . The time scales show 1 and 5 milliseconds. In the diagram of the upper limb, electrodes 1 and 2 are ring electrodes used for stimulation, 3 and 4 are the electrodes over the ulnar nerve distal to the cuff, and 5 and 6 are the electrodes over the ulnar nerve proximal to the cuff. An earth connexion was made at the plate placed on the forearm at $E$. Records $A, B, C$, and $D$ were taken respectively before the cuff was applied, five minutes and 25 minutes after the cuff was applied, and 2.5 minutes after release of the cuff. 
Fig. 8b.-The heights of the waves of the action potentials distal and proximal to the cuff are plotted, and also the ratio of the proximal to the distal height, expressed as a percentage.

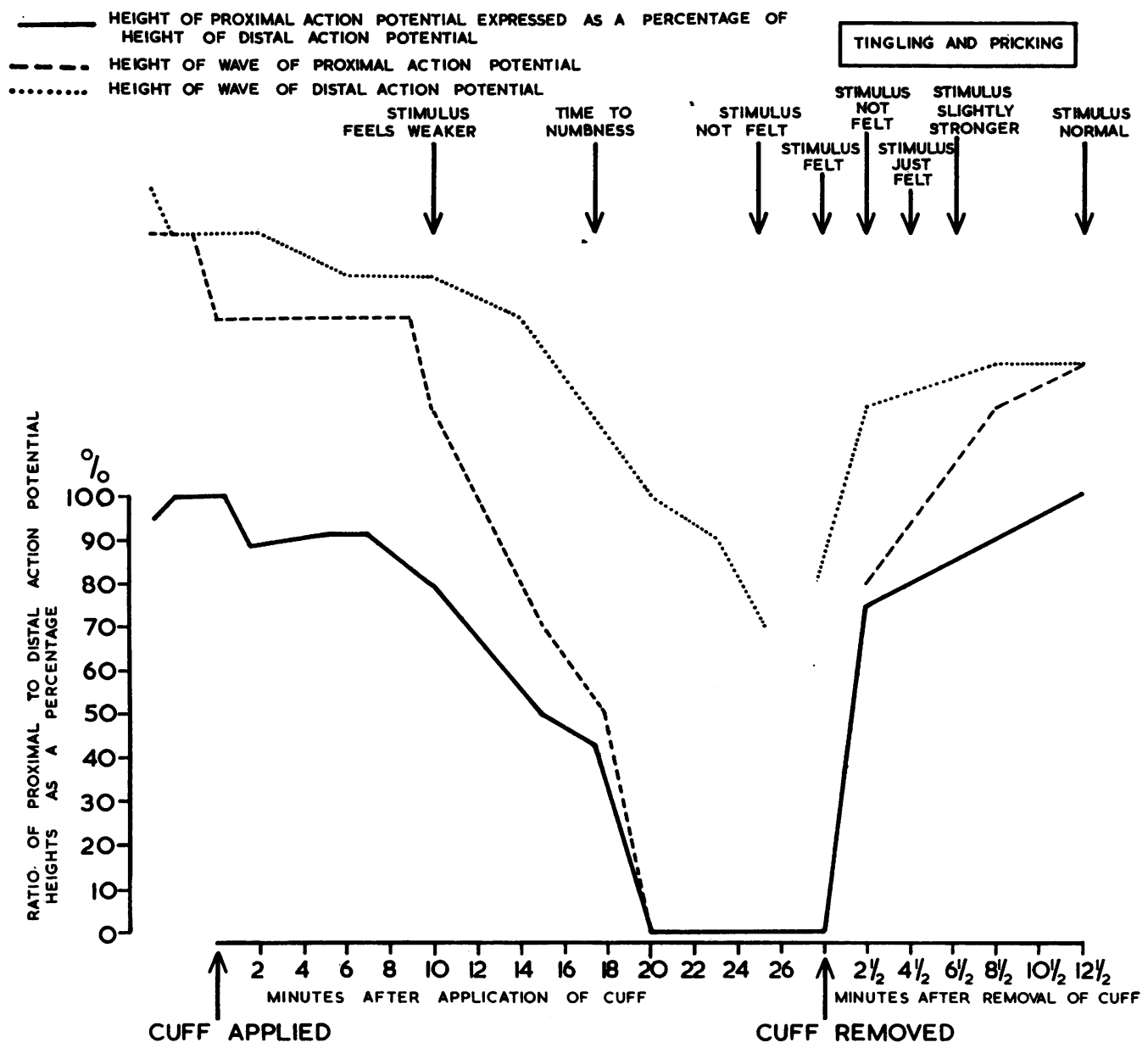

Thus, the question to be decided is whether the normal pattern of impulses needed for sensation fails to arrive at the central nervous system owing to being blocked in the peripheral nerve, or whether this normal pattern does arrive and is disturbed within the central nervous system owing to the concomitant arrival of impulses spontaneously generated in the same nerve.

Experiments were performed to find out if the impulses derived from electrical stimulation were arriving at the central nervous system and yet were not giving rise to sensation at a time when paraesthesiae were being experienced. The following experiment was carried out with the help of Dr. G. D. Dawson and Dr. R. W. Gilliatt, the latter on this occasion being the subject.

Experiment 8 (Figs. 8a and 8b). -The arrangement of electrodes has been described in the section on "Methods"; it is illustrated in Fig. 8a. The action potentials produced by stimulating the fourth and fifth digits were recorded distal and proximal to the cuff, placed over the elbow. In Fig. $8 \mathrm{~b}$ are plotted the heights of the proximal and distal action potentials, expressed as a percentage. During the early post-ischaemic period, at 2.5 minutes after release of the circulation to the limb, the subject was unable to feel the stimulus; in fact, in this actual experiment, he asked Dr. Dawson to look at the stimulating electrodes, as he felt sure that they must have slipped off. At this time it can be seen from Fig. 8a $(D)$ that there was a large action potential proximal to the cuff. It may then be concluded that the impulses were passing into the central nervous system. This inability to feel the stimulus occurred at a time when the size of the compound action potential proximal to the cuff was $75 \%$ of that distal to the cuff. During the ischaemic phase, when the compound action potential proximal to the cuff was 
$75 \%$ to $80 \%$ of that distal to the cuff, the stimulus had been clearly felt; and it had been felt even when the proximal action potential was too small to be recorded. Further, within the first halfminute of restoration of the circulation, before the paraesthesiae had begun, the stimulus was felt; and at this stage the size of the action potential both distal and proximal to the cuff was less than what it was later, when the stimulus causing this action potential failed to give rise to any sensation.

This and other similar experiments show that when paraesthesiae are severe, strong stimulation within the cutaneous distribution of a nerve does not arouse a conscious sensation, although the volley due to this stimulation can be shown to pass along the nerve towards the central nervous system. Thus, of the two hypotheses proposed, the evidence suggests that the one is true which states that there is a disturbance in the central nervous system which gives rise to the failure to feel the test stimulus. Experiments such as Experiment 8 also show that, during the periods of spontaneous generation of impulses, the whole nerve is capable of conducting single synchronous volleys. While this holds true for electrical stimulation, it would appear to be justified to suppose that the same applies to the impulses set up by stimulation with more natural forms of stimuli, as with a hair, with the two-point discriminator, and with a needle.

That the masking of sensations arising in the periphery is caused by some central disturbance might also be deduced from two of the experiments reported above, namely, Experiments 6 and 7. In these experiments, during the post-ischaemic paraesthesiae the sensation of pain was not aroused when appropriate stimulation was applied. It is known that the nerve fibres concerned in the mediation of pain sensibility do not discharge spontaneously during the post-ischaemic period. Thus a peripheral mechanism does not account for the obliteration of sensation also in these two experiments.

The impulses arising spontaneously do not have to arouse a conscious sensation before obliteration of the sensation aroused by peripheral stimulation takes place. This is seen when an experiment is carried out on the lower limb; often in this limb no ischaemic tingling is experienced. At the time when such paraesthesiae occur in the upper limb, namely,
Fig. 9.-Cuff on arm. Stimulus, hair of strength 1.

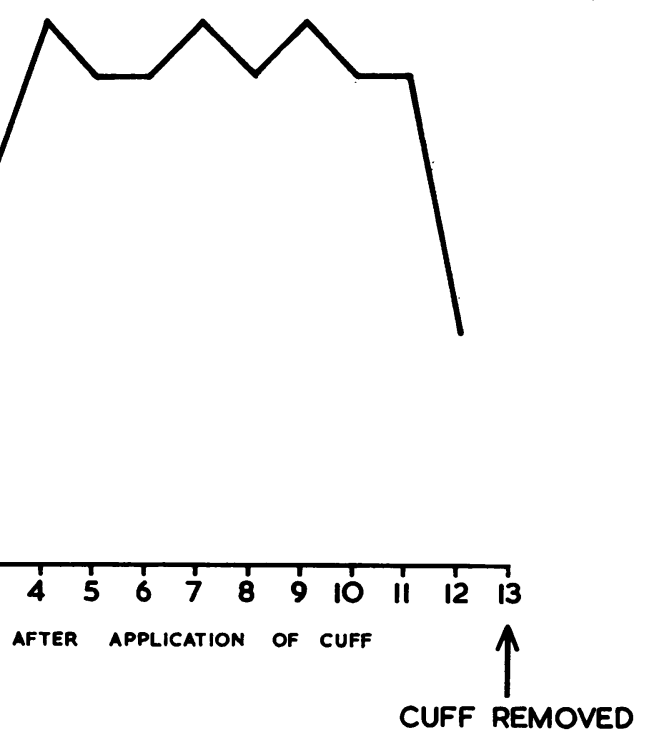

about one to three minutes after occlusion of the circulation, there is in the lower limb the usual inability to feel light touches. Thus, this sensation is masked, even though no paraesthesiae are being felt. The same thing can occur with certain lesions affecting sensory pathways in the central nervous system. This is shown, for instance, by the following case.

Experiment 9 (Fig. 9).-Mr. W. suffered from a thrombosis of the left posterior inferior cerebellar artery. In addition to the loss of thermal and painful sensibility on the right side of the body, there was a very slight abnormality of tactile sensibility of the left upper limb. This was sufficient to prevent the sensation of ischaemic tingling, for slight lesions of the tactile pathway prevent the appearance of ischaemic tingling.

The stimulus was the nylon No. 3 hair, strength 1 , applied to the left index finger, just proximal to the nail. Fifty applications were felt before the cuff was applied in the standard position. Although there was no ischaemic tingling, there was diminution in the number of responses to touch occurring at the time when ischaemic tingling is usually experienced (see Fig. 9).

In this patient interruption of the sensory pathway was within the medulla oblongata; it is probable that the lesion at this level was the cause of his failure to experience ischaemic tingling. Though in this patient the impulses usually causing tingling apparently did not enter consciousness, nevertheless, they could produce an effect which obscured the sensation due to the test stimulation. This effect presumably was not being caused below the lesion in the medulla oblongata. 


\section{Discussion}

It is usually considered that numbness results from a diminution in the number of conducting fibres. However, following the work of Gilliatt and Wilson and that reported here, it is apparent that numbness may also accompany an increased excitability of the larger nerve fibres. It may be due to such fibres spontaneously firing off impulses into the central nervous system; the reception of these impulses impairs the perception of peripheral stimulation, and this can occur even though the spontaneously arising impulses are giving rise to no sensation. Thus, numbness can be due to an inadequate number of conducting fibres; it can also be due to a large number of fibres firing off spontaneously when they are in a hyperexcitable state. No doubt this kind of numbness occurs under abnormal biochemical conditions. The changes occurring soon after the onset of vascular occlusion and very soon after the release from that occlusion are known to be the same as those produced by an increase in $p H$ or a decrease in calcium ion concentration. Robinson (1955) has studied sensation in patients with tetany. He found that when a cuff is placed on the upper limbs of these patients numbness occurs twice during the period of occlusion; first it occurs at the time of ischaemic tingling, which is marked in such cases, and second when the nerve fibres cease to conduct, which they do after very short periods of occlusion of the circulation.

It has always seemed curious to me how patients tend to use the terms " numb" and "tingling" indiscriminately. That there is a reduced ability to feel peripheral, stimulation during periods of paraesthesiae is now clear, and the more marked the paraesthesiae, the more definite the disturbance of peripheral sensation will be.

This is not to imply that all numbness is associated with tingling; in fact, much of the numbness of pathological conditions is similar to the velvety numbness that occurs after 20 minutes or so of an occluding cuff on the limb of normal subjects; this form of numbness is due to the failure to conduct of large numbers of the fibres.

The occurrence of these central factors in the obscuring of sensation must not lead one to account for all of the sensations associated with the periods of paraesthesiae as being due to central events. Many of these phenomena are most easily accounted for on the basis of an interaction in the peripheral nerves between orthodromic impulses and spontaneously arising antidromic and orthodromic impulses. For instance, during the period of postischaemic paraesthesiae, there may be a short period during which each adequate stimulation with a hair causes a feeling like a buzz continuing till the next application of the stimulus. This is most easily explained on the basis of reiterative firing and prolonged after-discharge during a phase of greatly increased excitability. When excitability is further increased, then each peripheral stimulus will cause a little volley of paraesthesiae; it is likely that the impulses due to peripheral stimulation, on reaching the region of spontaneous firing of impulses, set off groups of reiteratively firing impulses; thus they increase the spontaneous discharge. This is probably the explanation of the well-known fact that pins and needles can be increased by rubbing or pressing upon the part in which they are felt. A similar explanation probably underlies the fact that just before paraesthesiae appear spontaneously, they can be induced by rubbing or pressing the parts in which they are about to be felt.

It is surprising to find from experiments such as Experiment 8 that single synchronous volleys can get through to the central nervous system, and, under certain circumstances, give rise to no sensation. It is even more surprising to anyone who has felt the stimulus used for it is only just bearable; if it were made stronger, it would be impossible to relax the limb. If such a massive stimulus can be obliterated during the period of post-ischaemic paraesthesiae, it is likely that the same occurs for lighter and more usual kinds of stimulation.

Normal sensation depends on more complicated events than those arranged in Experiment 8. Such a single volley derived from a short stimulus, applied midway on the course of a nerve through the skin, is an artificial affair, and unlike the natural stimulation from everyday stimuli applied to end-organs. Impulses, in fact, are never single but come in battalions; trains of impulses are set off when the stimulus is applied and trains are set off when the stimulus is removed, and both are followed by an after-discharge. To alter sensation, it may be enough to alter the after-discharge or it may be enough to prolong the relatively refractory period of some fibres, so that alternate impulses, for instance, do not get through. It is unknown how much interference with the normal pattern of impulses arriving at the neurological substratum of sensation can be tolerated without there being an interference with normal sensation.

\section{Summary}

During the periods of ischaemic and post-ischaemic paraesthesiae, stimuli applied to the territory of the nerve spontaneously discharging are either not felt or they are felt in a diminished degree.

This interference with the perception of stimuli is enough to cause a real disturbance of sensation, 
and it is described as "numbness" by those experiencing it.

The forms of sensibility obliterated during these periods are touch, pressure, and pain. (Thermal sensations have not been investigated.)

It was found that this " numbness" is not due to a failure of the impulses to reach the central nervous system; it is, therefore, due to some process taking place within the central nervous system.

It is concluded that numbness is not only due to a large number of fibres ceasing to conduct impulses, but it can also be due to fibres being hyperexcitable and firing off spontaneously; in such a case peripheral stimulation is not felt.

I take this opportunity of thanking Dr. George Dawson and Dr. Roger Gilliatt who performed a great many of the experiments with me and helped throughout by useful discussions, and Dr. E. Arnold Carmichael for his interest and the opportunities he provided for doing this work.

\section{REFERENCES}

Bazett, H. C. and McGlone, B. (1929). Amer. J. Physiol., 90. 278. , (1932). Arch. Neurol. Psychiat. (Chicago), 28, 71. Bishop, G. H. (1943). J. Neurophysiol., 6, 361. (1944). Ibid., 7,71 and 185 .

Cobb, W., and Marshall, J. (1954). J. Neurol. Neurosurg. Psychiat.. $17,183$.

Dawson, G. D. (1956). J. Physiol., (Lond.), 131, 436.

and Scott, J. M. (1949). J. Neurol. Neurosurg. Psychiat., 12. 259.

Gilliatt, R. W. (1955). Ibid., 18, 145. and Wilson, T. G. (1953). Lancet, 2, 595

- (1954). J. Neurol. Neurosurg. Psychiat., 17, 104.

Kugelberg, E. (1944). Acta physiol. scand., 8, Suppl. 24 1948) Arch. Neurol Psychiat (Chicago) 60, 140.

and Cobb, W. (1951). J. Neurol. Neurosurg. Psychiat., 14, 88. Lehmann, J. E. (1937). Amer. J. Physiol., 119, 111

Lehmann, J. E. (1937). Amer. J. Physiol., 119, 11931). Heart. 16, 1. Lewis, T., Pickering, G. W., and Rothschild, P. (1931). Heart. (1953). Ibid., 16, 19.

Merrington, W. R., and Nathan, P. W. (1949). Ibid., 12, 1.

Poole, E. W. (1956a). Ibid., 19, 148

-(1956b). Ibid., 19, 281.

(1957). Ibid., 20, 225.

Robinson, P. K. (1955). Neurology, 5, 461.

Sinclair, D. C. (1948). J. Neurophysiol., 11, 75. 